\title{
The influence of dichotical fusion on the redundant signals effect, localization performance, and the mismatch negativity
}

\author{
Anja Fiedler • Hannes Schröter • Verena C. Seibold • \\ Rolf Ulrich
}

Published online: 16 November 2010

(C) Psychonomic Society, Inc. 2010

\begin{abstract}
In two experiments, each including a simple reaction time (RT) task, a localization task, and a passive oddball paradigm, the physical similarity between two dichotically presented auditory stimuli was manipulated. In both experiments, a redundant signals effect (RSE), high localization performance, and a reliable mismatch negativity (MMN) was observed for largely differing stimuli, suggesting that these are coded separately in auditory memory. In contrast, no RSE and a localization rate close to chance level (experiment 1) or at chance (experiment 2) were observed for stimuli differing to a lesser degree. Crucially, for such stimuli a small (experiment 1) or no (experiment 2) MMN were observed. These MMN results indicate that such stimuli tend to fuse into a single percept and that this fusion occurs rather early within information processing.
\end{abstract}

Keywords Event related potential · Mismatch negativity · Redundant signals effect $\cdot$ Fusion

Usually participants respond faster to the presentation of two redundant signals than to the presentation of a single one (e.g., Bucur, Allen, Sanders, Ruthruff, \& Murphy, 2005; Corballis, 2002; Diederich \& Colonius, 2004; Fischer \& Miller, 2008; Miller, 1982; Mordkoff \& Yantis, 1991, 1993). This gain in reaction time (RT) is known as redundant signals effect (RSE). Recent studies (Schröter,

A. Fiedler $\cdot$ H. Schröter $\cdot$ V. C. Seibold $\cdot$ R. Ulrich

Cognitive and Biological Psychology, University of Tübingen,

Tübingen, Germany

\author{
A. Fiedler $(\triangle)$ \\ Psychologisches Institut, University of Tübingen, \\ Friedrichstrasse 21, \\ 72072, Tübingen, Germany \\ e-mail: anja.fiedler@uni-tuebingen.de
}

Frei, Ulrich, \& Miller, 2009; Schröter, Ulrich, \& Miller, 2007), however, provided evidence that the RSE in the auditory modality does not depend on the mere number of physical signals, but rather on the number of percepts associated with those signals.

In a series of simple RT experiments by Schröter et al. (2007), participants were asked to respond to the onsets of auditory stimuli that were presented via headphones to the left ear, the right ear, or both ears. In redundant trials, pure tones of either identical frequency (experiment 1a: e.g., $500 \mathrm{~Hz}$ left; $500 \mathrm{~Hz}$ right) or of different frequency (experiment 1b: e.g., $500 \mathrm{~Hz}$ left; $700 \mathrm{~Hz}$ right) were presented. In a further experiment, a pure tone and white noise were presented to the left ear and to the right ear, respectively. Schröter et al. observed a reliable RSE only in the latter experiment. The authors attributed this pattern of results to a specific characteristic of the auditory system. Accordingly, two identical pure tones (diotical presentation) will be fused into a single percept which is internalized in the middle of the head (e.g., Leakey, Sayers, \& Cherry, 1958; Odenthal, 1961, 1963; Ward, 1970). In contrast, a pure tone and white noise are too different from each other in order to be fused into a single percept and thus, will be represented as two separate percepts. Consequently, these two separate percepts will result in an RSE.

Crucially, no reliable RSE was observed in the former experiment when two pure tones of different frequency were presented dichotically. This suggests that these tones might be similar enough to be fused into a single percept analogously to pure tones of identical frequency. To gain more evidence for this assumption, Schröter et al. (2007) asked participants to localize one of two dichotically presented auditory stimuli. Localization performance was nearly perfect when a pure tone and white noise were presented. In contrast, localization performance was largely 
deteriorated when pure tones of different frequency (i.e., $500 \mathrm{~Hz}+700 \mathrm{~Hz}$ ) were presented. Thus, in this condition participants were not able to retrieve the information about the single tone locations in each trial. These results support the notion that the dichotically presented pure tones of $500 \mathrm{~Hz}$ and $700 \mathrm{~Hz}$ were similar enough to be fused into a single percept, albeit they were not similar enough to be fused perfectly in each trial or for each participant, because localization performance was still above chance level. Taken together, the results of Schröter et al. (2007; see also Schröter et al., 2009) suggest that the occurrence of an RSE depends on the number of percepts rather than on the number of physical stimuli or on the number of activated receptors. Furthermore, whether and to which extent two redundant auditory stimuli fuse into a single percept seems to depend on the physical similarity between these stimuli.

The present study assessed the mismatch negativity (MMN) in addition to behavioral data to examine the locus of fusion within information processing. The MMN is an event-related brain potential (ERP) that reflects the automatic detection of deviances from a regular auditory input (for reviews, see Kujala, Tervaniemi, \& Schröger, 2007; Näätänen, Paavilainen, Rinne, \& Alho, 2007; Schröger, 1998, 2007). Specifically, the MMN can be reliably observed in an oddball paradigm in which a frequent standard sound and an infrequent deviant sound are presented in random succession. The MMN is typically derived by computing the difference between the ERPs elicited by the deviant and those elicited by the standard. Usually it exhibits a frontocentral distribution and peaks between 100 and $250 \mathrm{~ms}$ from the onset of deviation.

It is widely believed that the repetitive presentation of the standard sound creates a short-term memory trace in the auditory system (e.g., Näätänen, 1995; for an alternative view see Jääskeläinen et al., 2004). An MMN emerges when a mismatch detector is activated by a deviant sound that does not match this memory trace (e.g., Näätänen, 2001; Näätänen et al., 2007; Woldorff, Hackley, \& Hillyard, 1991). Following this assumption, the MMN can be interpreted as an indicator for a mismatch detection process that operates on the comparison between an incoming sound and the sensory memory trace that was built up in response to a repetitive standard stimulus.

There is evidence that attention directed towards the auditory oddball stimuli can influence the MMN (for a recent overview see Sussman, 2007). Thereby, the MMN in response to intensity deviants is more susceptible to attentional influences (e.g., Woldorff et al., 1991; Woldorff, Hillyard, Gallen, Hampson, \& Bloom, 1998) than the MMN in response to frequency deviants, which is rather insensitive to attentional manipulation (e.g., Näätänen, Tiitinen, Jiang, \& Alho, 1993; Paavilainen, Tiitinen, Alho, \& Näätänen, 1993). Nevertheless, the MMN can still index pre-attentive mismatch detection, at least when participants' attention is detracted from the oddball by a concurrent secondary task.

Although the MMN has been extensively investigated, the relationship between MMN parameters and auditory input is not always clear-cut. For example, the existing literature does not provide conclusive evidence whether the automatic comparison between standards and deviants is based on separate auditory features (e.g., Deacon, Nousak, Pilotti, Ritter, \& Yang, 1998; Gomes, Ritter, \& Vaughan, 1995; Molholm, Gomes, Lobosco, Deacon, \& Ritter, 2004; Schairer, Gould, \& Pousson, 2001) or on an integrated representation of the combined stimulus features (e.g., Grimm \& Schröger, 2007; Ritter, Sussman, \& Molholm, 2000; Sussman, Gomes, Nousak, Ritter, \& Vaughan, 1998; Takegata \& Morotomi, 1999). Although several accounts have been proposed to provide an integrative explanation of some of the seemingly contradictory MMN results (e.g., the hybrid memory model by Ritter, Deacon, Gomes, Javitt, \& Vaughan, 1995; the zip-metaphor mechanism by Grimm \& Schröger, 2007; the regularity-violation hypothesis, see e.g., Winkler, 2007), it is not well understood under which circumstances the MMN process operates on integrated representations and under which ones on separate stimulus features.

Furthermore, it has previously been assumed that the size of the MMN amplitude is associated with participants' ability to discriminate between standards and deviants (for a review, see Kujala et al., 2007). There is now a growing body of evidence, however, that an MMN can occur even when participants are unable to discriminate between a standard and a deviant (Alho \& Sinervo, 1997; Allen, Kraus, \& Bradlow, 2000; Neuloh \& Curio, 2004; van Zuijen, Simoens, Paavilainen, Näätänen, \& Tervaniemi, 2006). For example, Allen et al. (2000) determined individual discrimination thresholds for each participant and compared the MMN for suprathreshold stimulus differences as well as for subthreshold stimulus differences. In both threshold conditions, reliable MMNs were observed that did not significantly differ from each other. Therefore, Allen et al. argued that the MMN indexes pre-attentive processes that are not related to conscious perception. Crucially, these results suggest that chance performance at a behavioral level does not imply that brain processes are insensitive to differences between standards and deviants.

This high sensitivity of the MMN to indicate stimulus differences is central for the purpose of the present study. Specifically, it allows us to address the question at which level of information processing the hypothesized fusion of two dichotically presented pure tones occurs. More precisely, we use the MMN to examine whether such fusion takes place before or after pre-attentive sensory memory stages, or sensory memory for short. Accordingly, given a 
standard assignment of tones to the two ears (e.g., a lowpitched tone to the left ear and a high-pitched tone to the right ear), a deviant change in this assignment (e.g., a switched presentation of the two tones to the two ears, that is, a high-pitched tone to the left ear and a low-pitched tone to the right ear) should not be detected if these tones were already coded as a single auditory event within sensory memory. The location information should be lost and thus, the difference between standards and deviants should not be detected. Consequently, no MMN should emerge. In contrast, if the tones were fused into a single percept but are still represented separately at the level of sensory memory, a deviant change of tone locations should produce an MMN because the mismatch detector is supposed to operate on this unfused information within sensory memory (for reviews, see Kujala et al., 2007; Näätänen et al., 2007; Schröger, 1998, 2007). The results of two previous studies support this latter assumption. In the study by Praamstra and Stegeman (1992) pure tones of $440 \mathrm{~Hz}$ and $880 \mathrm{~Hz}$ were used. A reliable MMN was observed when these dichotically presented pure tones were occasionally switched between ears. The same result was observed by Deouell, Bentin, \& Giard (1998) for pure tones of 740 and $932 \mathrm{~Hz}$. Although both studies did not assess discrimination performance at a behavioral level, they nevertheless strongly indicate that two dichotically presented stimuli of different frequency can be represented separately in auditory memory.

Taken together, the present study investigated two main issues within two experiments. The first aim was to examine the influence of the physical difference between dichotically presented auditory stimuli on the degree of fusion indicated by two behavioral indicators, namely the RSE and localization performance. We hypothesized that the degree of fusion of two dichotically presented auditory stimuli depends on their physical similarity. If similar stimuli successfully fuse at least in the vast majority of trials, no RSE should be observed and localization performance should be about chance level. On the other hand, no fusion should occur for dissimilar stimuli resulting in a reliable RSE and a virtually perfect localization performance. The second aim was to investigate the locus of the fusion process for dichotically presented similar stimuli with the help of the MMN. If such stimuli are already represented as single event in auditory memory without their individual location information, a deviant change of tone location should be undetected and no MMN should be observed. Alternatively, if the two tones are separately represented in sensory memory, a deviant change of location should be detected and this should result in an MMN. Such an outcome would suggest that the two tones are fused after sensory memory processes. Since dissimilar stimuli are expected to be perceived as separate percepts and thus should be represented separately within sensory memory including information about their individual location, a deviant change of these locations should be detected, thus resulting in a reliable MMN. ${ }^{1}$

Experiment 1 employed the same stimuli and conditions that were used by Schröter et al. (2007), namely a tone+ tone condition $(500 \mathrm{~Hz}+700 \mathrm{~Hz})$ and a tone + noise condition (either $500 \mathrm{~Hz}$ or $700 \mathrm{~Hz}+$ white noise). Experiment 2 employed pure tones which differed either slightly $(300 \mathrm{~Hz}+321 \mathrm{~Hz})$ or largely $(300 \mathrm{~Hz}+831 \mathrm{~Hz})$ in frequency. In both experiments, each subject participated in three sub-tasks designed to assess the RSE (simple RT task), localization performance (2AFC task), and the MMN (passive oddball paradigm).

\section{Experiment 1}

Experiment 1 lasted four hours including the application of electrodes. The participants started with the MMN experiment, proceeded to the RSE experiment, and completed the session by performing the localization experiment. This order was chosen due to the increasing attentional demand of the experiments. In the following, we will first present the methods and results of the simple RT task (experiment 1a) and the localization task (experiment 1b) as these were set up to replicate the results of Schröter et al. (2007). Then, we will present the methods and results of the crucial MMN experiment (experiment 1c). In experiment 1 , we compared two conditions. In the tone + tone condition two pure tones of different frequency $(500 \mathrm{~Hz}+700 \mathrm{~Hz})$ were dichotically presented. In the tone + noise condition a pure tone (either $500 \mathrm{~Hz}$ or $700 \mathrm{~Hz}$ ) and white noise were presented dichotically. We expected to replicate the results of Schröter et al. (2007) in the RSE and the localization experiment. Specifically, we expected to observe an RSE as well as almost perfect localization performance in the tone+noise

\footnotetext{
${ }^{1}$ It should be noted that the traditional interpretation of the MMN as reflecting a sensory-memory trace mismatch has recently been challenged. According to some authors (e.g., Winkler, 2007), the auditory system develops neural models of the auditory environment. Regularities of incoming sounds as well as their inter-relationships are coded within these models. They are predictive in nature and thus serve as an important prerequisite for the detection and separation of auditory objects. Accordingly, the MMN is elicited when a deviant sound violates the regularities predicted by these neural models and thus the MMN can rely on auditory sensory information as well as rather abstract and categorical information. However, what is important for the present study is that according to both views a lack to find a reliable MMN due to a fusion process would indicate that neither the deviation in sensory input nor a violation of possible "abstract" rules has been detected. This, however, is also compatible with the idea that the fusion of dichotically presented auditory stimuli of high physical similarity is already eminent at the sensory memory stage.
} 
condition. In contrast, the two tones in the tone + tone condition were expected to fuse into a single percept at least in the majority of trials. Thus, no RSE as well as worse localization performance should result in this condition.

Of main interest is the outcome of the MMN experiment. As the stimuli in the tone+noise condition were expected to be separately perceived and thus should also be represented separately within sensory memory including their location information, a deviant change of these locations should be detected. Therefore, a reliable MMN should emerge in this condition. In contrast, the stimuli in the tone+tone condition were expected to fuse into a single percept and, thereby to lose their individual location information. In this condition, two alternative outcomes are conceivable. If the mismatch detector operates on the fused representation of stimuli, a deviant change of tone location should be undetected. Consequently, no MMN should be observed. Such a result would suggest an early locus of the fusion process. Alternatively, if the two tones are separately represented, a deviant change of location should be detected and result in an MMN. Such a result would suggest that the two tones are fused after sensory memory processes.

\section{Experiment 1a: simple RT task}

In experiment $1 \mathrm{a}$, we assessed the RSE in a simple RT task. Participants had to respond to the onsets of auditory stimuli that were presented either to the left ear, to the right ear, or to both ears. In the tone+tone condition, these stimuli were pure tones (a $500-\mathrm{Hz}$ tone presented to one ear and a $700-\mathrm{Hz}$ tone presented to the other ear). In the tone + noise condition, auditory stimuli consisted of a pure tone presented to one ear and white noise presented to the other ear. In order to avoid interstimulus contingencies (see Mordkoff \& Yantis, 1991) that might promote stimulus detection in redundant target trials, the probability of a stimulus presented to one ear was independent of whether the second stimulus was presented to the other ear. Specifically, this probability was $p=.6$. Accordingly, a response was required in $84 \%$ of all trials and no response was required in the remaining catch trials in which no auditory stimuli were presented.

Method

Participants Twenty-four students of the University of Tübingen (22 female, mean age: 21.8 years; range: $19-$ 30 years) with a mean handedness score (Oldfield, 1971) of .75 (range: $.41-1.0$ ) took part in the experiment. They received either partial fulfillment of a course requirement or payment (28 euros) for their participation in the complete session. All experiments were performed in accordance with the ethical standards laid out in the 1964 Declaration of Helsinki. Written informed consent was obtained from all participants. The data of two additional participants had to be excluded from analysis due to a high error rate in experiment $1 \mathrm{a}$ and due to noisy data in experiment $1 \mathrm{c}$, respectively.

Apparatus and stimuli All experiments took place in a darkened and soundproof booth. The presentation of stimuli was controlled by Experimental Run Time System (ERTS; Berisoft, Frankfurt am Main, Germany) running on a DOS computer. The auditory stimuli were either white noise or pure tones with a frequency of $500 \mathrm{~Hz}$ and $700 \mathrm{~Hz}$, respectively. They were of $300-\mathrm{ms}$ duration each and presented via headphones (Sony MDR-CD570) with an intensity of $60 \mathrm{~dB}$ SPL. All auditory stimuli were filtered externally by a Butterworth filter. The waveform started with a zero crossing at stimulus onset. To avoid abrupt stimulus onsets (offsets), stimulus intensity was increased (decreased) exponentially resulting in a rise (decay) time of $50 \mathrm{~ms}$ when a tone was presented (terminated). The white noise was generated by MATLAB with a bandwidth of $0-44.1 \mathrm{kHz}$ and it had the same intensity and envelope as the pure tones. However, it was also filtered externally by the Butterworth filter, reducing the signal strength for frequencies above the cutoff frequency of $1.25 \mathrm{kHz}$ by $3 \mathrm{~dB}$ per octave. Therefore, the presented stimulus was a mixture of white noise (below the cutoff-frequency) and pink noise (above the cutoff-frequency). A white plus sign $\left(0.5^{\circ} \times 0.5^{\circ}\right.$ of visual angle) was used as warning signal as well as fixation cross. It was presented centrally against a blue background on a standard computer screen with a viewing distance of $60 \mathrm{~cm}$.

Feedback was given by presenting the phrases "Richtig!" (Correct!) following correct responses, "Zu langsam!" (Too slow!) following too slow responses, or "Reagiere nur auf das Anschalten eines auditiven Reizes!" (Do only respond to the onset of an auditory stimulus!) following false alarms. Half of the participants responded with their left index finger and the left "Shift-Key" of a 101/102-standard keyboard and the other half with their right index finger and the right "Shift-Key".

Procedure A single experimental block consisted of 150 trials. Thereof, 126 trials included the presentation of an auditory stimulus (go trial) whereas in 24 trials no auditory stimulus was presented (catch trial). An auditory stimulus was presented exclusively to the left or to the right ear on 36 go trials each. On 54 go trials, auditory stimuli were presented simultaneously to the two ears. In go trials, participants were asked to respond quickly and accurately with a single key press as soon as they detected any 
auditory stimulus irrespective of tone location. In catch trials, participants were instructed to withhold their response. The order of trial type was randomized within each experimental block.

Half of the participants first received 17 practice trials followed by an experimental block in the tone+tone condition. Afterwards they received 17 practice trials followed by an experimental block in the tone+noise condition. For the other half of the participants, this assignment was reversed. For a given participant, the assignment of the two pure tones to the two ears (tone+tone condition) as well as the assignment of the pure tone and the white noise to the two ears (tone+noise condition) was kept constant throughout the experiment. This assignment was counterbalanced across participants.

Each trial started with the presentation of the fixation cross which remained on the screen until a response was registered or for a maximal period of 1,600 ms. On go trials, auditory stimuli were presented $600 \mathrm{~ms}$ after the onset of the fixation cross for $300 \mathrm{~ms}$. On catch trials, no auditory stimulus was presented until the trial had terminated. Written feedback was provided for $1,500 \mathrm{~ms}$, followed by an intertrial interval of $900 \mathrm{~ms}$. After a run of 50 trials, participants could take a short rest and received feedback about their performance in the previous run of trials. The next run of trials started after participants pressed a key.

\section{Results and discussion}

Trials with RT shorter than $110 \mathrm{~ms}(0.3 \%$ on average, including anticipations) or longer than $800 \mathrm{~ms}(0.8 \%$ on average, including misses) were considered outliers and thus excluded from data analysis. ${ }^{2}$ The false alarm rate in catch trials was low $(0.7 \%$ on average). Redundant signals effects were assessed by comparing the mean RT of the faster of the two single-stimulus conditions (faster singlestimulus condition) against the mean of the redundant condition, because an RT advantage for the redundant condition over the average of the two single-stimulus conditions could be an artifact of averaging across participants who detected the left stimulus earlier and other participants who detected the right stimulus earlier (cf. Biederman \& Checkosky, 1970). Figure 1 depicts the mean RT on go trials in experiment 1a.

An ANOVA with the within-subjects factors of redundancy (faster single vs. redundant) and stimulus condition (tone+tone vs. tone+noise) was performed on

\footnotetext{
${ }^{2}$ None of the analyses revealed qualitatively different results when outliers were included.
}

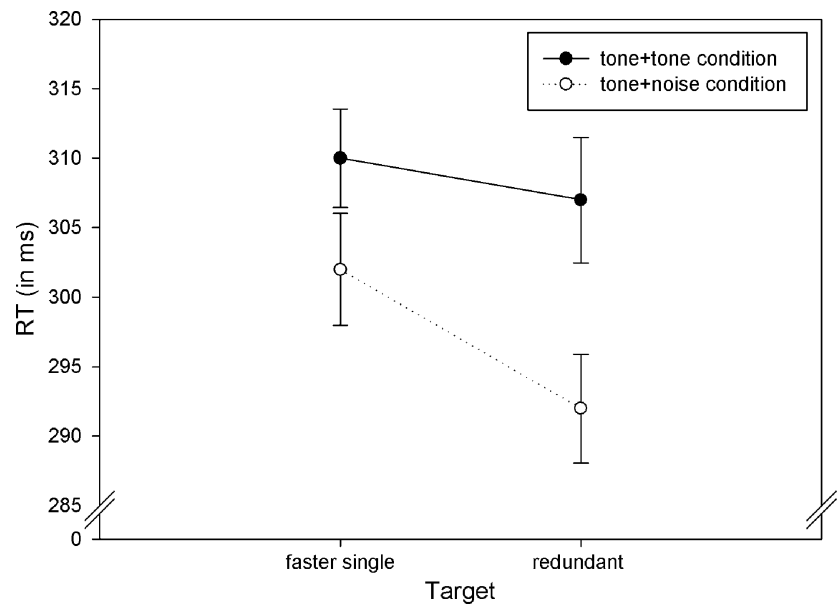

Fig. 1 Mean reaction time for go trials in experiment 1a as a function of redundancy and stimulus condition. Error bars show the mean \pm standard error using the algorithm for within-subject designs recommended by Cousineau (2005)

RT. This ANOVA revealed a significant effect of redundancy, $F(1,23)=7.54, M S E=118.24, p<.05$. Mean RT was significantly shorter for redundant stimuli (300 ms) than for the faster single stimulus (306 ms). The factor stimulus condition failed to reach significance, $F(1,23)=2.17, M S E=1,353.6, p>.05$. Most importantly, the interaction between both factors reached significance, $F(1,23)=4.28, M S E=73.77, p=.05$. Additional $t$ tests revealed that RT for redundant stimuli (307 ms) was not significantly shorter than RT to the faster single stimulus $(310 \mathrm{~ms})$ in the tone+tone condition, $t(23)=0.81, p>.05$. In contrast, RT was significantly shorter for redundant stimuli $(292 \mathrm{~ms})$ than for the faster single stimulus $(302 \mathrm{~ms})$ in the tone+noise condition, $t(23)=3.76, p<.005 .^{3}$ In conclusion, experiment $1 \mathrm{a}$ replicated the major findings of Schröter et al. (2007). A significant RSE was observed in the tone+noise condition but not in the tone+tone condition. This result supports the notion that dichotically presented pure tones differing in frequency can be fused into a single percept. To test this assumption, we assessed the localization performance in experiment $1 b$.

\footnotetext{
${ }^{3}$ To assess potential redundancy effects at the distributional level, Vicentized RT distributions were computed for the sum of the single stimulus and the redundant stimuli condition used to test the race model inequality (see Miller, 1982; Ulrich, Miller, \& Schröter, 2007). The present data did not violate this inequality, neither in the tone +tone nor in the tone+noise condition (all $p s>.05$ ). In contrast to the present study, Schröter et al. (2007) reported a small but significant violation of the race model inequality in the tone+noise condition. The respective experiment of Schröter et al. (2007) included more experimental trials per condition than the present experiment. Therefore, it is likely that the power of the present study was insufficient to detect a violation of the inequality.
} 


\section{Experiment 1b: 2AFC localization task}

In experiment $1 \mathrm{~b}$, we asked the participants to localize a specific target pure tone simultaneously presented with either a pure tone of different frequency (tone + tone condition) or white noise (tone+noise condition). We expected again to replicate the findings of Schröter et al. (2007). Specifically, the stimuli in the tone + noise condition should be perceived as separate percepts including their individual location information. Therefore, we expected an almost perfect performance in this condition. In contrast, since the two tones in the tone+tone condition were expected to fuse into a single percept, participants should not be able to indicate which ear receives the high-pitched and which one receives the low-pitched tone. Therefore, we expected localization performance to be close to chance level in the tone+tone condition.

\section{Method}

Participants The same participants as in the previous experiment took part in experiment $1 \mathrm{~b}$.

Apparatus and stimuli The apparatus, the auditory stimuli, and the fixation cross were identical to experiment 1 . No feedback, however, was provided to the participant.

Procedure The beginning of each trial was again indicated by a fixation cross. After $600 \mathrm{~ms}$, the predefined target stimulus, a pure tone, was presented to one ear while the other ear was simultaneously stimulated by either another pure tone of different frequency or white noise. Participants had to indicate in which ear they had perceived the target by pressing the corresponding key (the left Shift-Key if they perceived the target on the left ear and the right Shift-Key if they perceived the target on the right ear). They were instructed to be as accurate as possible and therefore obtained sufficient time for their response (max. 5,000 ms). Furthermore, they were instructed to guess the location of the target whenever they were unsure about its location.

Overall, there were two experimental blocks with 200 trials each. Half of the participants localized the $500-\mathrm{Hz}$ tone in the first block, and the $700-\mathrm{Hz}$ tone in the second block. This assignment was reversed for the other half of participants. Twenty-five practice trials preceded each experimental block in order to familiarize participants with the task. Participants could take a short rest after a run of 50 trials. In half of all trials, the target tone was presented to the left ear, and in the other half of trials it was presented to the right ear. The non-target stimulus was a pure tone in $50 \%$ of all trials and white noise in the remaining trials. Presentation order of these trials was randomized.

\section{Results and discussion}

An ANOVA with the within-subjects factors target location (left ear vs. right ear) and stimulus condition (tone + tone vs. tone + noise) was performed on the arc-sine transformed percent of correct responses which comply better with the assumption of normally distributed error components (Winer, 1971). Most importantly, the ANOVA revealed a significant effect of stimulus condition, $F(1,23)=130.82$, $M S E=0.15, p<.001$. As expected, localization performance was much higher in the tone+noise condition (96.6\%) than in the tone+tone condition (67.5\%), though localization performance was above chance level in the latter condition, $t(23)=4.8, \quad p<.001$. The results of experiment $1 \mathrm{~b}$ are in line with those of Schröter et al. (2007), strengthening the notion that the two dichotically presented pure tones were fused into a single percept in the majority of trials (e.g., in $65 \%$ of all trials after correcting for guessing; see Macmillan \& Creelman, 2005, p. 172). However, the finding that localization performance was not exactly at chance level indicates that fusion was imperfect in some of the trials and/or participants. ${ }^{4}$

\section{Experiment 1c: passive oddball paradigm}

In experiment $1 \mathrm{c}$, we employed the MMN to examine whether this fusion took place before or after sensory memory processes are engaged. As stated in the Introduction, if a deviant change of stimulus locations elicits an MMN in the tone+tone condition, this would suggest that the two tones are still coded separately within sensory memory. Therefore, such a finding would argue for a late locus of the fusion process. In contrast, the absence of an MMN would suggest that the two tones are already represented as a single auditory event within sensory memory and thus would indicate an early locus of the fusion process. As a control, we again included the tone+noise condition. Here, we expected to obtain an MMN, since deviant location changes of the two auditory stimuli represented separately within sensory memory should activate the mismatch detector.

Method

Participants The same participants as in the previous experiments took part in experiment $1 \mathrm{c}$.

\footnotetext{
${ }^{4}$ We included a short discussion about the nature of the fusion process in the General Discussion.
} 
Apparatus and stimuli The apparatus and the stimuli were identical to experiment $1 \mathrm{~b}$, however, there was no fixation cross in this experiment.

Procedure Participants watched a silent movie (Buster Keaton; ca. $90 \mathrm{~min}$ ) on the monitor while auditory stimuli were presented binaurally via headphones. Participants were instructed to attend to the movie and to ignore the auditory stimuli. Auditory stimuli were presented in eight blocks of 260 stimuli each with an interstimulus interval of 2,200 ms.

For a given participant, the assignment of auditory stimuli to the left and the right ear was identical to experiment 1a. For deviant stimuli the locations of the two auditory stimuli were reversed (Deouell et al., 1998; Praamstra \& Stegeman, 1992) and these deviants were presented with $p=.1$. For example, for a given participant the standard consisted of the $700-\mathrm{Hz}$ tone presented to the left ear and the $500-\mathrm{Hz}$ tone presented to the right ear. For the deviant stimulus, the $700-\mathrm{Hz}$ tone was presented to the right ear and the $500-\mathrm{Hz}$ tone to the left ear. Half of the participants first received four blocks in the tone+tone condition followed by four blocks in the tone+noise condition. This assignment was reversed for the remaining participants. After each block, participants could take a short rest.

To establish an internal memory representation of the standard, ten standards were presented at the beginning of each block. These stimuli were excluded from data analysis. Standards and deviants were presented pseudorandomized, that is, at least four standards preceded a deviant.

Electrophysiological recordings Electroencephalographic (EEG) and electroocular (EOG) activity (both bandpasses: $0.05-50 \mathrm{~Hz}$ ) was continuously recorded using a Neuroscan amplifier system. EEG was recorded from midline electrodes $\mathrm{Fpz}, \mathrm{Fz}, \mathrm{Cz}, \mathrm{Pz}$, over the left hemisphere from electrodes $\mathrm{F} 3, \mathrm{C} 3, \mathrm{~T} 3$, and $\mathrm{O} 1$, over the right hemisphere from electrodes $\mathrm{F} 4, \mathrm{C} 4, \mathrm{~T} 4$, and $\mathrm{O} 2$ and from both mastoids (Tp9, Tp10) using an Easycap-System (EASYCAP GmbH, Herrsching, Germany). The tip of the nose served as reference and AF8 as ground. Horizontal EOG was registered from the left and right outer canthi and the vertical EOG from above and below the left eye. Electrode impedances were kept below $5 \mathrm{k} \Omega$ at all scalp and face sites. All electrophysiological signals were digitized at $250 \mathrm{~Hz}$.

Data analysis The analysis epoch started $140 \mathrm{~ms}$ prior to stimulus onset and lasted for a total duration of $540 \mathrm{~ms}$. Standards following a deviant were excluded from data analysis, as well as epochs in which the EEG or EOG change exceeded $\pm 100 \mu \mathrm{V}$. Vertical and horizontal eye movements were corrected using the algorithm described by Gratton, Coles, \& Donchin (1983). EEG and EOG signals were averaged time-locked to the onset of the auditory stimuli, separately for experimental conditions. For each participant and each condition, MMN waveform was derived by subtracting the average ERPs elicited by deviants and by standards. Each grand average of these difference waveforms was filtered offline (30-Hz low-pass filter). For each participant, individual MMN mean amplitudes were calculated in a time window of $40 \mathrm{~ms}$ around the peak latency of the grand average MMN (Schröger, 1998). MMN peak latencies were measured and analyzed by applying the jackknife procedure (Miller, Patterson, \& Ulrich, 1998; Ulrich \& Miller, 2001). Student's $t$ test was used to determine the presence of an MMN by comparing the mean amplitude against zero. $T$ test was also used for comparing MMN peak latencies and mean amplitudes between the two conditions.

\section{Results and discussion}

Figure 2 shows the grand-average ERPs for standards and deviants as well as the corresponding difference waveform at $\mathrm{Fz}$ and at the pooled mastoids for each condition (but see also Fig. 3 for the deviant-minus-standard difference waveforms at $\mathrm{Fz}$ ). The MMN waveforms peaked at $138 \mathrm{~ms}$ from stimulus onset in the tone+tone condition and at $121 \mathrm{~ms}$ in the tone+noise condition; this difference in MMN peak latency did not differ between the tone +tone and the tone+noise conditions, $t_{c}(23)=0.47, p>.05$. Mean MMN amplitude of the difference waveforms was more pronounced $(-1.119 \mu \mathrm{V})$ in the tone+noise condition than in the tone + tone condition $(-0.439 \mu \mathrm{V}), t(23)=2.57$, $p<.05$. Mean MMN amplitude was significantly different from zero in the tone + noise condition, $t(23)=5.37$, $p<.001$. This result clearly indicates that the location deviant has been detected in the tone+noise condition. Most importantly, however, a reliable MMN was also observed in the tone + tone condition, $t(23)=2.30, p<.05$, suggesting that at least in some trials the mismatch system detected the location deviant in this condition.

To sum up, experiment 1 replicated the main behavioral findings of Schröter et al.'s (2007) study. Accordingly, a pure tone and white noise incorporate a large physical difference, resulting in two redundant and clearly distinguishable percepts. Thus, an RSE could be observed, localization performance was high and a reliable MMN was elicited. However, in the tone+tone condition no RSE was observed. The assumption that the lack of finding an RSE in this condition might be due to a fusion of these 


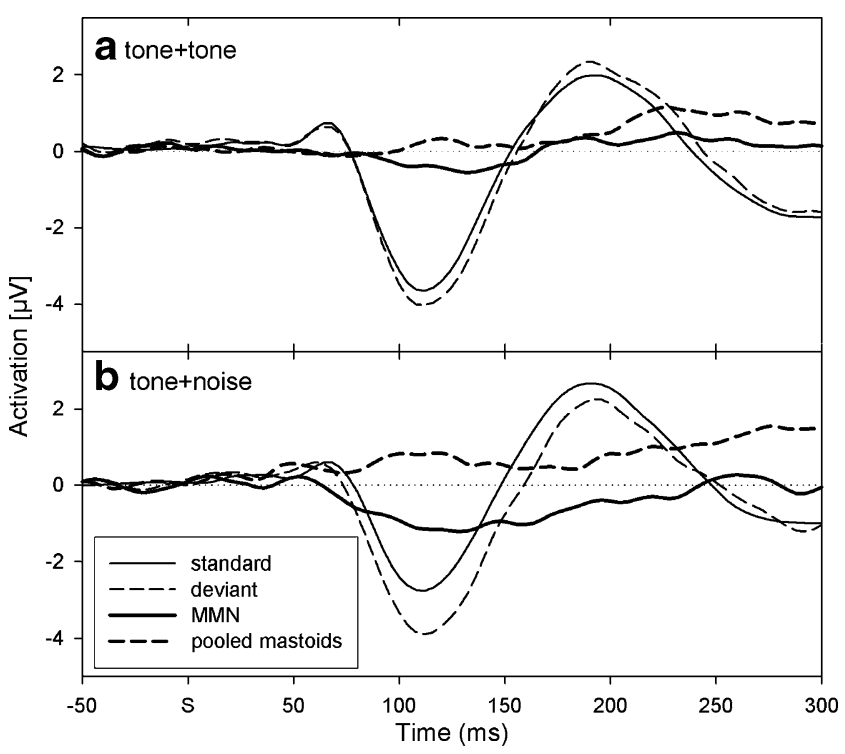

Fig. 2 Grand-averaged standard (thin-solid) and deviant (thin-dashed) ERPs, and deviant-minus-standard difference waveforms (thick-solid) recorded at $\mathrm{Fz}$ for the tone+tone (upper panel) and tone+noise (lower panel) conditions in experiment $1 \mathrm{c}$. Additionally, the deviant-minusstandard difference waveform recorded at the pooled mastoids is depicted (thick-dashed) for the two conditions. The data were referenced against the nose electrode. $S$ indicates stimulus onset

tones was confirmed by the corresponding localization performance that was rather poor. However, as localization performance was still above chance level, it is conceivable that the fusion was not perfect. Hence, the $500-\mathrm{Hz}$ and $700-$ $\mathrm{Hz}$ tones were similar enough to be successfully fused in most of the cases but not similar enough to be fused perfectly in all trials. This assumption is in line with the present MMN results. The small but reliable MMN amplitude in the tone+tone condition suggests that the

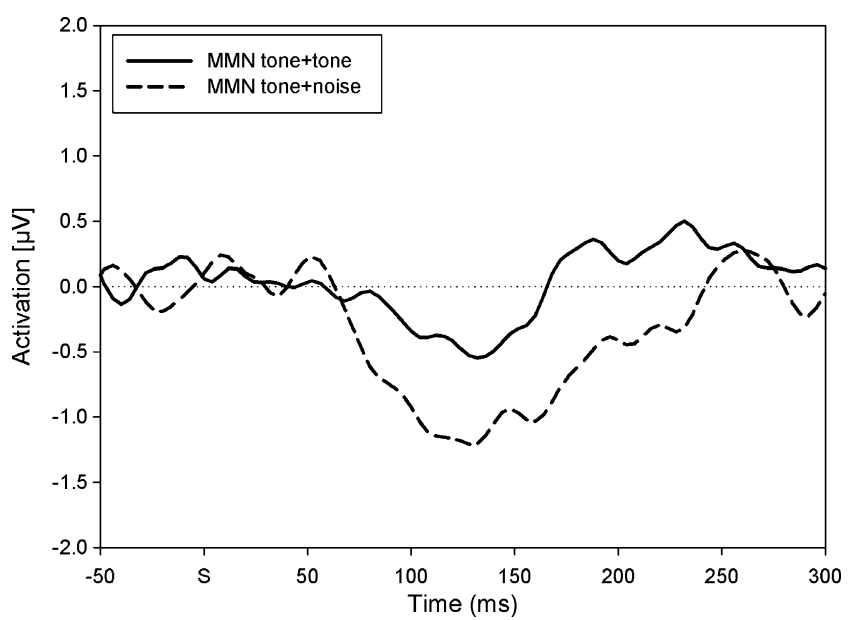

Fig. 3 Grand-average difference waves (deviant minus standard) recorded at $\mathrm{Fz}$ for the tone+tone (solid) and the tone+noise (dashed) conditions in experiment $1 \mathrm{c}$. The data were referenced against the nose electrode. $S$ indicates stimulus onset location deviant was detected in some cases, suggesting that the fusion process was not always perfect.

However, there is an alternative explanation for the present MMN results. Accordingly, two mismatch detectors might exist that work independently for the left and right channels. The deviation in one single ear is larger in the tone+noise condition (e.g., left ear standard: $500 \mathrm{~Hz}$, left ear deviant: white noise) than in the tone+tone condition (e.g., left ear standard: $500 \mathrm{~Hz}$, left ear deviant: $700 \mathrm{~Hz}$ ). Previous studies suggest that an increase in the magnitude of deviance might result in an increase in MMN amplitude (e.g., Novitzki, Tervaniemi, Huotilainen, \& Näätänen, 2004; for an alternative view, see also Horvath et al., 2008). Thus, the relative difference in MMN amplitudes for the tone+tone and the tone+noise condition could be simply due to the greater physical distance between standard and deviant (i.e., for a single ear) in the tone+noise condition. Whereas the localization performance strongly suggest that the pure tones in the tone + tone condition are fused although not perfectly, the relative small MMN in this condition might be due to a fusion or, alternatively, to a relative small physical difference between standard and deviant in each ear.

To provide stronger evidence for the notion that the physical difference between dichotically presented auditory stimuli affects a fusion process of such stimuli we conducted a second experiment. On the one hand, the pure tones of $500 \mathrm{~Hz}$ and $700 \mathrm{~Hz}$ in experiment 1 did not result in a perfect fusion. Thus, for experiment 2, we designed new auditory stimuli and assessed the influence of their physical difference on the RSE, localization performance and the MMN. On the other hand, we incorporated additional control conditions in the passive oddball paradigm, in order to test whether the observed differences in MMN amplitudes between conditions are due to a fusion process or to the mere physical difference between standard and deviant for each single ear.

\section{Experiment 2}

In experiment 2, we dichotically presented two pairs of pure tones. In the small frequency distance condition, tone pairs consisted of a $300-\mathrm{Hz}$ tone and a $321-\mathrm{Hz}$ tone (with the relative difference equaling $7 \%$ and $6.5 \%$ for a standard of $300 \mathrm{~Hz}$ and $321 \mathrm{~Hz}$, respectively). Hence, in this condition the physical difference between tones was dramatically decreased in comparison with the tone+tone condition in experiment 1 . In line with our assumptions, this decrement in physical difference should enhance the fusion of these tones indicated by the absence of an RSE and a localization performance at chance level. Furthermore, we expected to observe no MMN in this condition given that the fusion process occurs rather early. 
In the large frequency distance condition, a second tone pair was used, consisting of a $300-\mathrm{Hz}$ and an $831-\mathrm{Hz}$ tone (relative difference equaling $177 \%$ and $64 \%$ for a standard of $300 \mathrm{~Hz}$ and $831 \mathrm{~Hz}$, respectively). In this condition, we increased the physical similarity between tones as compared to the tone+tone condition of experiment 1 . Thus, we expected that for this large frequency distance less fusion occurs than for the physically similar tones. Crucially, the physical difference between these tones was restricted to a mere frequency difference, whereas the pure tone and the white noise in experiment 1 differed on many aspects (e.g., spectral complexity, pitch information). Thus, on the one hand, due to the large frequency distance, we expected an RSE, a high localization performance, and a reliable MMN in this large frequency distance condition. On the other hand, the large frequency distance might not prevent fusion on each trial and thus results might be not as strong as for the tone+noise condition in experiment 1 .

In contrast to experiment 1 , the passive oddball task designed to record the MMN included two additional control conditions in order to test whether observed differences in MMN amplitudes between conditions are due to a fusion process or to the mere physical difference between standard and deviant for each single ear. In these conditions, which used the same stimuli as the experimental small and large distance conditions, tones were not presented dichotically but diotically (e.g., a 300-Hz pure tone was presented as standard simultaneously to both ears and a $321-\mathrm{Hz}$ pure tone was presented as deviant simultaneously to both ears). We will refer to these control conditions with diotical presentation as "Frequency-Oddball" whereas we will refer to the experimental conditions with dichotical presentation as "LocationOddball". Note that the presentation of a deviant will result in the same frequency change for a single ear (e.g., LocationOddball with small frequency distance: $300 \mathrm{~Hz}$ to the left ear and $321 \mathrm{~Hz}$ to the right ear changes to $321 \mathrm{~Hz}$ to the left ear and 300 to the right ear; Frequency-Oddball with small frequency distance: $300 \mathrm{~Hz}$ to both ears changes to $321 \mathrm{~Hz}$ to both ears).

We included the control conditions due to two reasons. First, the control conditions enabled us to check whether the absence of an MMN in the Location-Oddball condition might simply be the consequence of the small frequency distance used. Accordingly, if no MMN is observed for this frequency distance in the Location-Oddball condition, but a reliable MMN is elicited by the same frequency distance when embedded in a Frequency-Oddball condition, this allows for a more specific interpretation. Specifically, the presence of an MMN in the Frequency-Oddball condition would indicate that the small frequency distance itself is sufficient to elicit an MMN. Importantly, the absence of an MMN in the Location-Oddball condition would then provide evidence that the two tones are fused when presented dichotically. Second, even if an MMN occurs in both the Frequency-Oddball and the Location-Oddball conditions it is still important to know whether the MMN amplitudes in both conditions differ for tones with a small frequency distance. Specifically, the finding of no MMN amplitude difference would provide evidence for the view that two mismatch detectors operate independently for the left and right channels. Hence, differences in MMN mean amplitude between the small and the large frequency distance condition could then be due to differences between standard and deviant in each single ear.

In contrast, the finding of an MMN amplitude difference would provide evidence for the view that a single mismatch detector operates on a combined representation of the left and right channels, suggesting that differences between the small and the large frequency distance condition are due to a fusion process (for discussions see e.g., Deouell et al., 1998; McKenzie \& Barry, 2006; Praamstra \& Stegeman, 1992). The dissociation between these two possibilities is crucial for the interpretation of MMN amplitude differences between the small and the large frequency distance conditions, which could be either due to a fusion process or to the mere physical difference between standard and deviant in each ear. Though we were mainly interested in the comparison between the Location-Oddball and the Frequency-Oddball for the small frequency distance condition, we also included a Frequency-Oddball condition for the large frequency distance, which served as further control condition. Specifically, in line with previous studies (e. g., Horvath et al., 2008; Jacobsen \& Schröger, 2001), we expected to observe a reliable MMN for tones of large frequency distance also in the Frequency-Oddball condition.

Analogous to experiment 1, experiment 2 included an RSE, a localization, and an MMN experiment. The whole experiment lasted for $7.5 \mathrm{~h}$ and was therefore divided into two sessions. In the first session $(3 \mathrm{~h})$, participants completed one-half of experiment $2 \mathrm{c}$. In the second session, they completed the second half of experiment $2 \mathrm{c}$ and experiment $2 \mathrm{a}$ and $2 \mathrm{~b}$. The order of experiments was chosen for the same reasons as for experiment $1 \mathrm{a}-\mathrm{c}$. The two sessions were separated by 1-14 days.

\section{Experiment 2a: simple RT task}

Method

Participants A fresh sample of twenty-four students from the University of Tübingen (13 female, mean age: 25.5 years; range: $19-47$ years) participated in experiment 2a, with a mean handedness score (Oldfield, 1971) of .62 
(range: .68-1.0) for right-handed $(n=19)$ and .74 (range: .28-.87) for left-handed participants. They received either partial fulfillment of a course requirement or payment (52.50 euros). All experiments were performed in accordance with the ethical standards laid out in the 1964 Declaration of Helsinki. Written informed consent was obtained from all participants. The data of two additional participants had to be excluded from data analysis due to technical problems and noisy data, respectively.

Apparatus and stimuli The apparatus and stimuli were identical to experiment $1 \mathrm{a}$ with the following exceptions: The tone+tone condition was replaced by the small frequency distance condition. In this condition, a $300-\mathrm{Hz}$ tone was presented to one ear and a $321-\mathrm{Hz}$ tone was presented to the other ear. The tone+noise condition was replaced by the large frequency distance condition. Here, a $300-\mathrm{Hz}$ pure tone was presented to one ear and an $831-\mathrm{Hz}$ pure tone was presented to the other ear.

Procedure The procedure was identical to experiment 1a except for the number of trials that was increased to 200 trials per condition.

\section{Results and discussion}

Trials with RT shorter than $110 \mathrm{~ms}(0.4 \%$ on average, including anticipations) or longer than $800 \mathrm{~ms}(0.3 \%$ on average, including misses) were considered outliers and thus excluded from data analysis. The false alarm rate in catch trials was low $(0.2 \%$ on average). The RSE was assessed by comparing the mean RT of the faster of the two single-stimulus conditions (faster single-stimulus condition) against the mean of the redundant condition. Figure 4 depicts the mean RT on go trials in experiment 2 a.

An ANOVA with the within-subjects factors redundancy (faster single vs. redundant) and frequency distance (small vs. large) was performed on RT. This ANOVA revealed a significant effect of redundancy, $F(1$, $23)=4.87, M S E=147.41, p<.05$. Mean RT was significantly shorter for redundant stimuli $(333 \mathrm{~ms})$ than for the faster single stimulus (338 $\mathrm{ms})$. The factor frequency distance failed to reach significance, $F<1$. Most importantly, the interaction between both factors was significant, $F(1,23)=6.09, M S E=40.87, p<.05$. Additional $t$ tests revealed that RT for redundant stimuli (337 ms) was not significantly shorter than RT to the faster single stimulus (339 $\mathrm{ms}$ ) in the small frequency distance condition, $t(23)=0.80, p>.05$. In contrast, $\mathrm{RT}$ was significantly shorter for redundant stimuli (329 ms)

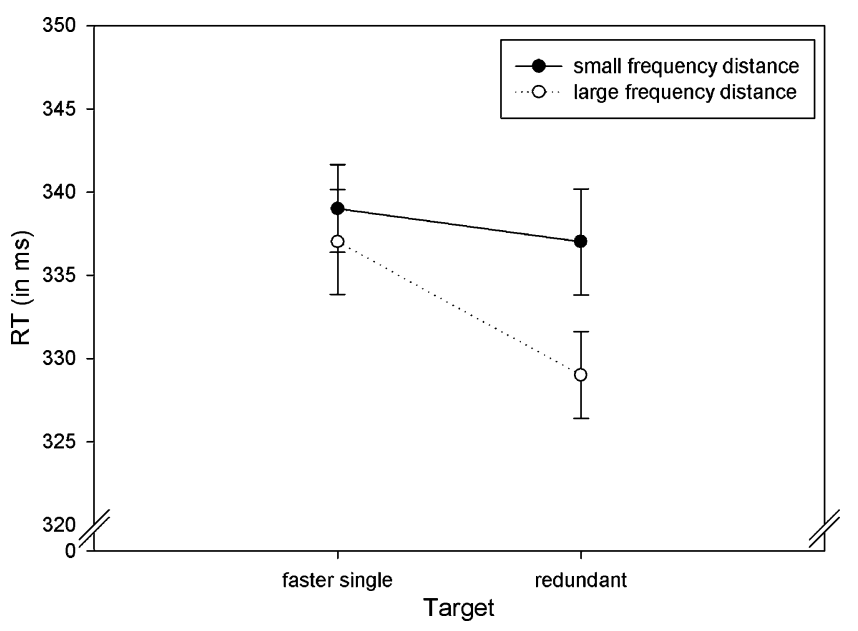

Fig. 4 Mean reaction time for go trials in experiment $2 \mathrm{a}$ as a function of redundancy and stimulus condition

than for the faster single stimulus $(337 \mathrm{~ms})$ in the large frequency distance condition, $t(23)=3.13, p=.005 .^{5}$

The results of experiment 2 a suggest that the occurrence of an RSE indeed depends on the frequency distance of dichotically presented pure tones. The absence of an RSE in the small frequency distance condition is in line with the findings of experiment $1 \mathrm{a}$ as well as the findings of the studies of Schröter et al. (2007, 2009). Furthermore, the observed RSE in the large distance condition provides first evidence that an RSE can occur for dichotically presented pure tones, provided that the frequency distance between these tones is considerably large. According to our hypothesis, the observed differences between both conditions can be explained by the number of percepts: Whereas dichotically presented pure tones of similar frequency are fused into a single percept, pure tones differing largely in frequency are represented as two separate percepts. To test this assumption, we conducted experiment $2 \mathrm{~b}$ in which we assessed the localization performance in these two conditions.

\section{Experiment 2b}

In experiment $2 b$, we examined the degree of fusion for the small and the large frequency distance conditions by measuring localization performance in both conditions. Based on the results of experiment $2 \mathrm{a}$, we expected localization performance to be at chance level in the small frequency distance condition whereas we expected high localization performance in the large frequency distance condition.

\footnotetext{
${ }^{5}$ As in experiment $1 \mathrm{a}$, the observed RSEs did not violate the race model inequality, neither in the small frequency nor in the large frequency condition (all $p s>.05$ ).
} 


\section{Method}

Participants The same participants as in experiment $2 \mathrm{a}$ took part in experiment $2 \mathrm{~b}$.

Apparatus and stimuli The apparatus and stimuli were identical to experiment $2 \mathrm{a}$.

Procedure. The procedure was identical to experiment $1 \mathrm{~b}$ except that experimental trials were increased to 280 trials per experimental condition.

\section{Results and discussion}

An ANOVA with the within-subjects factors target location (left ear vs. right ear) and frequency distance (small vs. large) was performed on the arc-sine transformed percent of correct responses (cf. Winer, 1971). Most importantly, the ANOVA revealed a significant effect of frequency distance, $F(1,23)=226.04, M S E=0.08, p<.001$. As expected, localization performance was much higher when frequency distance was large (86.7\%; range: $60-99 \%)$ than when it was small (50.1\%; range: $40-62 \%$ ). Localization performance was clearly above chance level for the large frequency distance condition, $t(23)=12.54, p<.001$, but at chance level in the small frequency distance condition, $t(23)=0.41, p>.05$.

The differential localization performance in the small and large frequency distance conditions strongly supports our hypothesis that the physical similarity of two dichotically presented stimuli determines whether they will fuse into a single percept. The at-chance level performance in the small frequency distance condition implies that participants could not retrieve the individual location information of the two tones suggesting that these tones were fused into a common representation. In contrast, localization performance in the large frequency distance condition was very high, albeit not as high as for the tone+noise condition in experiment $1 \mathrm{~b}$, suggesting that these tones were represented separately in the majority of trials (i.e., in $73.5 \%$ of all trials after correction for guessing).

\section{Experiment 2c}

In experiment $2 \mathrm{c}$, we again measured the $\mathrm{MMN}$ as an indicator for the automatic detection of a deviant location change of two dichotically presented pure tones. Basically, experiment $2 \mathrm{c}$ was analogous to experiment $1 \mathrm{c}$. In contrast to experiment $1 \mathrm{c}$, however, we compared the ERPs elicited by the stimuli used in experiment $2 a-b$, that is, dichotically presented pure tones that had either a small or a large frequency distance. Furthermore, as explained in the Introduction to experiment 2, we included two additional Frequency-Oddball conditions as a control. In these Frequency-Oddball conditions, the standard consisted of pure tones which, however, were not presented dichotically but diotically (e.g., a $300-\mathrm{Hz}$ pure tone simultaneously to the left and right ears). In $10 \%$ of all trials, these standard frequencies were replaced in both ears by identical deviant frequencies (e.g., small Frequency-Oddball: $321 \mathrm{~Hz}$ to both ears; large Frequency-Oddball: $831 \mathrm{~Hz}$ to both ears). Hence, and as outlined before, the physical difference between standard and deviant is exactly the same for the Frequency- and the Location-Oddball conditions for a single ear.

As mentioned in the Introduction of experiment 2, we expected a reliable MMN for the large frequency distance both in the Location-Oddball condition and in the Frequency-Oddball condition. Of main interest, however, is the outcome for the small frequency distance. Accordingly, if a reliable MMN is observed in the Frequencybut not in the Location-Oddball condition, this would indicate that the small frequency distance itself is sufficient to elicit an MMN. Importantly, such a pattern of results would indicate that the tones in the LocationOddball condition are fused into a single percept.

\section{Method}

Participants The same participants as in experiment $2 \mathrm{a}-\mathrm{b}$ took part in experiment $2 \mathrm{c}$.

Apparatus and stimuli The apparatus and stimuli were identical to experiment $1 \mathrm{c}$ with the following exceptions: In the small frequency distance conditions pure tones of $300 \mathrm{~Hz}$ and $321 \mathrm{~Hz}$ were presented. Furthermore, in the large frequency distance conditions tones of $300 \mathrm{~Hz}$ and $831 \mathrm{~Hz}$ were used. In the Location-Oddball conditions, these pure tones were presented dichotically, whereas in the Frequency-Oddball conditions they were presented diotically.

Procedure Experiment $2 \mathrm{c}$ was divided into two sessions. In each session four blocks of 500 stimuli each, with an interstimulus interval of $2,000 \mathrm{~ms}$, were presented binaurally via headphones. Throughout each block, participants watched a silent movie ("The Little Rascals"; ca. $160 \mathrm{~min}$ ). They were instructed to attend to the movie and to ignore the auditory stimuli. Furthermore, to assure that participants really attended to the movie and not to the auditory stimuli, they were told that they would be asked to answer questions about the movie, one question being asked after 
20 min each. After each block, participants could take a short rest.

In the Location-Oddball conditions, the assignment of auditory stimuli to the left and the right ear was identical to experiment $2 \mathrm{a}$ for a given participant. The deviant stimuli were created by reversing the locations of the two dichotically auditory stimuli. In the Frequency-Oddball conditions, identical frequencies were presented to both ears simultaneously. Deviants were created by changing the common standard frequency in both ears towards a common deviant frequency. In each condition standards and deviants $(p=.1)$ were presented pseudo-randomized, that is, at least four standards preceded a deviant. To establish an internal memory representation of the standard, 20 standards were presented at the beginning of each block. These stimuli were excluded from data analysis.

Electrophysiological recordings Both EEG and EOG were continuously recorded using a BIOSEMI amplifier system. EEG was recorded from 64 scalp electrodes. Additional recording sites were the tip of the nose (which served as reference later on) and both mastoids. Horizontal EOG was registered via electrodes at the left and right outer canthi and vertical EOG via electrodes above and below the left eye. EEG and EOG recordings were digitized at a sampling rate of $512 \mathrm{~Hz}$.

Data analysis The analysis epoch for each stimulus started $140 \mathrm{~ms}$ prior to stimulus onset and lasted for a total duration of $540 \mathrm{~ms}$. Epochs containing blinks were corrected using the adaptive artifact correction method in Brain Electromagnetic Source Analysis (BESA, Version 3.5.2) software (Ille, Berg, \& Scherg, 2002). Furthermore, standards following a deviant were excluded from data analysis, as well as epochs in which the EEG or EOG change exceeded $\pm 100 \mu \mathrm{V}$. EEG and EOG signals were averaged time-locked to the onset of the auditory stimuli, separately for each experimental condition. A pre-stimulus interval of $100 \mathrm{~ms}$ served as baseline.

For each participant and each condition, MMN was computed as the difference between the average ERP elicited by deviants and standards. Each grand average of these difference waveforms was filtered offline (with a 0.5$\mathrm{Hz}$ high-pass filter and a $30-\mathrm{Hz}$ low-pass filter). For each participant, individual MMN mean amplitudes were calculated in a time window of $40 \mathrm{~ms}$ around the peak latency of the grand average MMN (Schröger, 1998). MMN peak latencies were again measured and analyzed at the time point when negativity was maximal in the difference waveforms derived by the jackknife procedure (Miller et al., 1998; Ulrich \& Miller, 2001). For all four conditions, the presence of an MMN was determined by comparing the mean amplitudes against zero by means of Student's $t$ test.
Differences in peak latency and mean amplitude between conditions were assessed by a repeated measures ANOVA with the within-subjects factors oddball condition (frequency vs. location) and frequency distance (small vs. large).

\section{Results and discussion}

Figure 5 shows the grand-average ERPs for standards and deviants as well as the corresponding difference waveforms at $\mathrm{Fz}$ and at the pooled mastoids for each condition (compare also Fig. 6 for the deviant-minus-standard difference waveforms at Fz). In the Frequency-Oddball condition, statistically reliable MMNs were observed for both the large $(-1.014 \mu \mathrm{V}), t(23)=4.43, p<.001$, and the small frequency distance $(-0.545 \mu \mathrm{V}), t(23)=2.20$, $p<.05$. In contrast, in the Location-Oddball condition a reliable MMN was observed only for the large frequency distance $(-1.303 \mu \mathrm{V}), t(23)=4.04, p<.005$, whereas no reliable MMN was observed for the small frequency distance $(-0.177 \mu \mathrm{V}), t(23)=0.47, p>.05$.

The ANOVA for mean MMN amplitude revealed a significant effect of frequency distance, $F(1,23)=6.05$, $M S E=2.52, p<.05$, reflecting larger mean amplitudes for the large $(-1.158 \mu \mathrm{V})$ than for the small frequency distance conditions $(-0.361 \mu \mathrm{V})$. Neither the effect of oddball condition $(F<1)$ nor the interaction of oddball condition and frequency distance were significant, $F(1,23)=1.16$, $M S E=2.22, p>.05$.

Because there was no significant MMN signal in the Location-Oddball condition with the small frequency distance, no peak latency could be determined for this condition. Therefore, a single-factor ANOVA with the factor condition (Frequency-Oddball with small frequency distance, Frequency-Oddball with large frequency distance, Location-Oddball with large frequency distance) was performed. This ANOVA revealed a significant effect of condition, $F_{c}(1,23)=4.96, M S E=2,785.6, p<.05$. Contrasts using the Scheffé procedure (critical value: $38.5 \mathrm{~ms}, p<.05$ ) revealed that MMN peak latency was longer in the Frequency-Oddball condition with small frequency distance (199 ms) than in both the FrequencyOddball condition with large frequency distance $(155 \mathrm{~ms})$ and the Location-Oddball condition with large frequency distance $(160 \mathrm{~ms})$. Peak latency did not differ significantly in the two latter conditions $(p>.05)$.

We observed a reliable MMN for stimuli with a small frequency distance only in the Frequency-Oddball condition, but not in the Location-Oddball condition. Thus, one might argue that both conditions differ from each other, because in one condition an MMN was observed whereas 
Fig. 5 Grand-averaged standard (thin-solid) and deviant (thin-dashed) ERPs, and deviant-minus-standard difference waveforms (thicksolid) recorded at $\mathrm{Fz}$ for the four conditions: Frequency-Oddball with small frequency distance (upper left), Frequency-Oddball with large frequency distance (lower left), Location-Oddball with small frequency distance (upper right), and Location-

Oddball with large frequency distance (lower right) in experiment 2c. Additionally, the deviant-minus-standard difference waveform recorded at the pooled mastoids is depicted (thick-dashed) for each condition. The data were referenced against the nose electrode. $S$ indicates stimulus onset

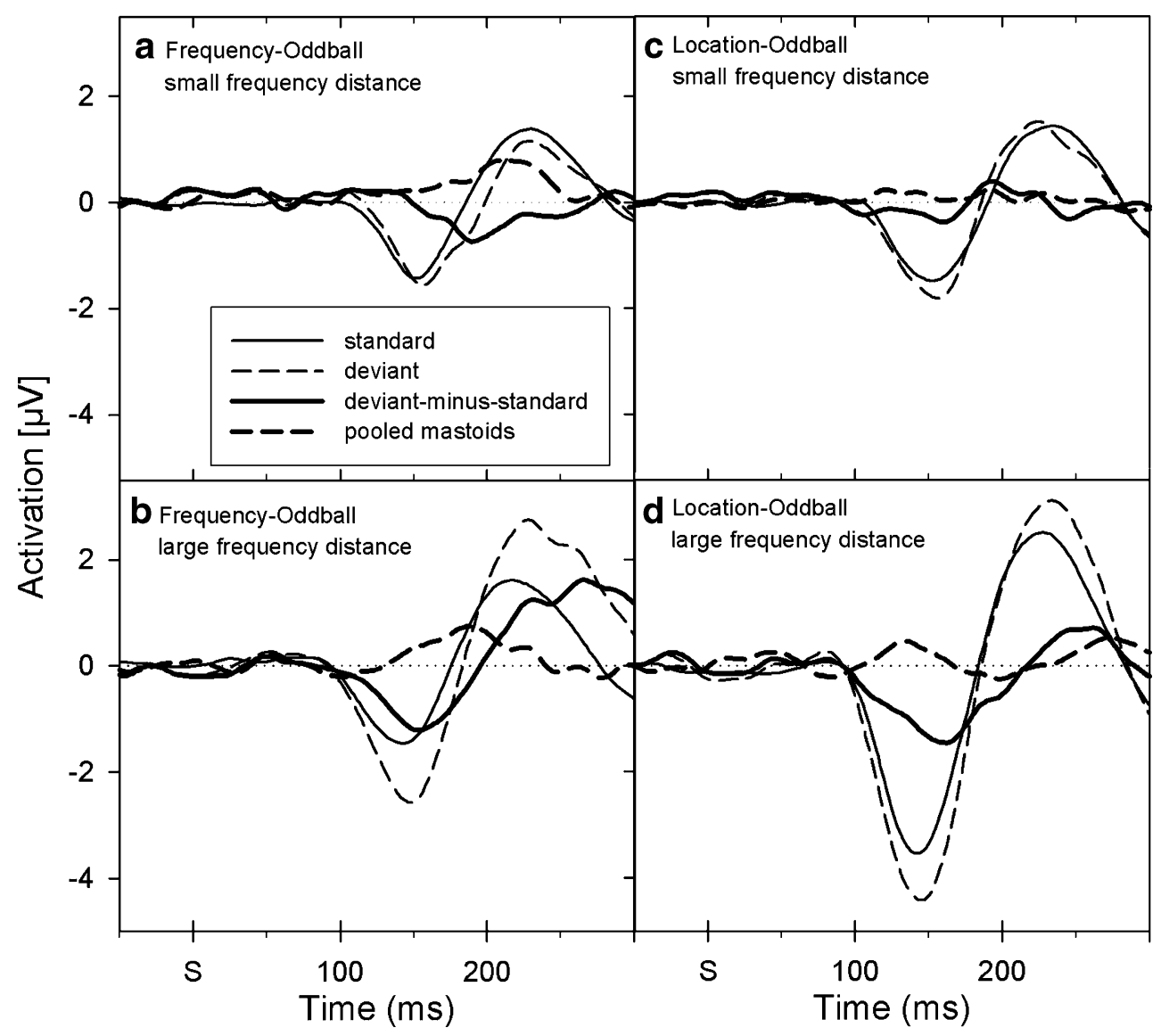

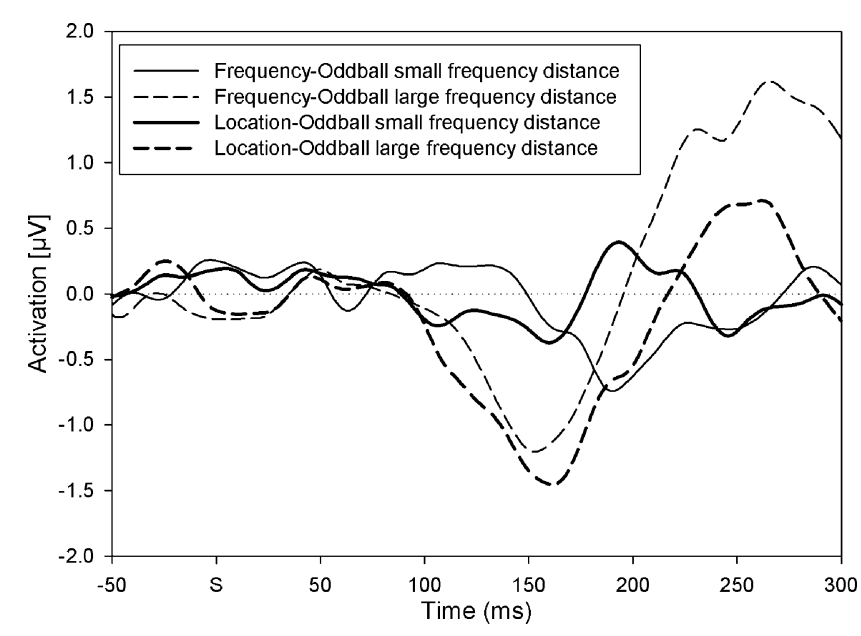

Fig. 6 Grand-average difference waves (deviant minus standard) in experiment $2 \mathrm{c}$ for the four conditions: Frequency-Oddball with small frequency distance (thin-solid), Frequency-Oddball with large frequency distance (thin-dashed), Location-Oddball with small frequency distance (thick-solid), and Location-Oddball with large frequency distance (thick-dashed). The data were recorded at Fz and referenced against the nose electrode. $S$ indicates stimulus onset in the other condition it was not. However, the comparison of the amplitudes for small frequency distance tones in the Location-Oddball and the Frequency-Oddball conditions did not yield significant differences. Nevertheless, visual inspection of the deviant-minus-standard difference waves in the two conditions (see Fig. 6) revealed that the timecourse clearly differed between the two conditions. Therefore, a moving window technique was employed to compare the difference waves of these two conditions. To this end, mean amplitudes were calculated at $\mathrm{Fz}$ for each condition in seven 25-ms-windows starting at $50 \mathrm{~ms}$ after stimulus onset. These amplitudes were analyzed with a 7 (seven time windows of $25 \mathrm{~ms}$ each) $\times 2$ (Location-Oddball vs. Frequency-Oddball) ANOVA. Neither the factor oddball nor the factor time window was significant (both $F_{\text {s }}<1$ ). However, there was a significant interaction between these two factors, $F(1,23)=3.62, M S E=0.72$, $p<.05$. Additional $t$ tests revealed that the amplitudes of the difference waves in the Frequency-Oddball and the Location-Oddball condition differed significantly from each other only in the sixth time window of 175 $200 \mathrm{~ms}, t(23)=2.12, p<.05$, and in the seventh time window of $200-225 \mathrm{~ms}, t(23)=2.98, p<.05$. This additional analysis provides evidence for our interpretation that for stimuli with small frequency distance the 
absolute amplitude difference between the LocationOddball and the Frequency-Oddball condition observed in the first five time windows (i.e., between $50 \mathrm{~ms}$ to $175 \mathrm{~ms}$ after stimulus onset) was not systematic, but due to noise. Crucially, the only significant differences in amplitudes were observed when the MMN in the Frequency-Oddball condition reached its peak. Taking these findings into account, it seems reasonable to conclude that an MMN was present in the FrequencyOddball but not in the Location-Oddball condition.

In summary, experiment $2 \mathrm{c}$ supports the notion that frequency distance (and thus the physical similarity) is crucial for the automatic detection of a deviant location change between two dichotically presented pure tones. Specifically, we observed a reliable MMN for dichotically presented pure tones with a large frequency distance. In contrast, no reliable MMN was observed for dichotically presented pure tones with a small frequency distance. This lack of a reliable MMN cannot be attributed to the frequency distance itself because the same frequency distance elicited a reliable MMN in the FrequencyOddball condition. Since the MMN is attributed to early auditory stimulus processing, that is, the detection of a mismatch at the level of sensory memory, the absence of an MMN in the Location-Oddball condition suggests that the two dichotically presented pure tones with small frequency distance were fused into a single percept at an early level of stimulus processing. Hence, together with the results observed in Experiments $2 \mathrm{a}-\mathrm{b}$, the results observed in the Location-Oddball condition again provide evidence for our hypothesis that the absence of an RSE for dichotically presented auditory stimuli can be attributed to a fusion of their perceptual representations. Moreover, the differential MMN results obtained for small frequency distance tones as compared to large frequency distance tones indicate that fusion seems to occur only for stimuli of similar frequency. In contrast, tones of very different frequencies do not seem to be fused, but to be coded as separate representations.

\section{General discussion}

The present study investigated the fusion of auditory stimuli and its effect on the RSE and localization performance. Specifically, we examined whether the physical difference of dichotically presented auditory stimuli influences the degree of fusion of these stimuli. In addition, we assessed the MMN as an indicator for the locus of such a fusion process. To this end, we conducted two main experiments in which we manipulated the physical difference of dichotically presented auditory stimuli by implementing a condition with stimuli of small frequency distance to each other and a condition with stimuli that differed largely. We hypothesized that physically similar stimuli are more likely to be fused than dissimilar stimuli and this should be reflected in the RSE and localization performance. In addition, we expected the electrophysiological results to provide evidence for an early locus of this fusion process.

The behavioral results of experiments 1 and 2 confirmed our hypothesis. Accordingly, in both experiments an RSE and high localization performance was observed for auditory stimuli, which differed largely. However, no RSE and a localization rate close to chance level (experiment 1) or at chance (experiment 2) were observed for pure tones that had a small frequency distance. This pattern of results supports the notion that the occurrence of the RSE depends on the number of percepts and thus on separate representations of the physical redundant stimuli.

The electrophysiological results suggest that the fusion occurs rather early in information processing. In experiment 1 as well as in experiment 2, a deviant location switch elicited a pronounced MMN for largely differing stimuli. In contrast, a small MMN was observed in the tone+tone condition of experiment 1 and no reliable MMN occurred for pure tones with a small frequency distance in experiment 2. Since the MMN occurs whenever a mismatch between auditory input and a sensory memory representation is detected, the lack of a reliable MMN for physical similar tones suggests that such stimuli are coded as a single event in sensory memory without their individual location information.

Comparing the behavioral and the electrophysiological data, the RSE seems to be a rather binary measure of the fusion process, whereas localization performance and MMN are more sensitive in assessing the degree of fusion. In experiments 1 and 2, an RSE reliably occurred for auditory stimuli that differed largely, but not for similar pure tones. The localization performance as well as the size of the MMN, however, indicated that the fusion process varies depending on the stimulus difference. Hence, pure tones of 500 and $700 \mathrm{~Hz}$ were similar enough to get fused in the majority of trials, thus preventing an RSE. It is important to mention though that their frequency distance was still large enough to prevent perfect fusion which was reflected by the above chance level localization performance and the presence of a small MMN. A further decrement of the frequency distance resulted in a perfect fusion of tones. Thus, the frequency distance between 300 and $321 \mathrm{~Hz}$ was small enough to diminish localization performance to chance level. Furthermore, for these stimuli a location switch remained undetected by the MMN system.

On the other side, increasing the frequency distance of two dichotically presented tones can prevent their fusion in the majority of trials. For pure tones of 300 and $831 \mathrm{~Hz}$, 
localization performance substantially improved and a reliable MMN was observed in response to a deviant location switch. The finding that fusion does not occur for stimuli of large physical difference is further supported by two other studies that observed an MMN for a deviant location switch of dichotically presented pure tones with a large frequency distance (Deouell et al., 1998; Praamstra \& Stegeman, 1992). Although these studies did neither assess discrimination performance nor included a condition with tones of small difference, their results nevertheless indicate that a rather large frequency difference can prevent fusion and thus a deviant location change of tones is detected by the MMN system.

Taken together, physical difference seems to influence the fusion of dichotically presented pure tones gradually. This gradual influence is indicated by location performance and MMN amplitude, which vary with the amount of physical difference. The occurrence of the RSE, however, is less sensitive to specific amounts of fusion, but rather seems to depend on a critical threshold of fusion.

An alternative interpretation of the present MMN results has been ruled out by the findings of experiment 2 . Accordingly, differences in MMN between conditions are probably not due to the physical difference between standard and deviant in each single ear but rather to the fusion process. More specifically, in experiment 2 each single ear received the same deviant frequency distance in the Frequency-Oddball and in the Location-Oddball condition. If the MMN detector operates on separate memory traces for the left and right ears, the MMN amplitude observed for small frequency distances should be equally large for the Frequency- and the Location-Oddball condition. This, however, was not the case. Hence, the results of experiment 2 are not in line with the idea that the MMN detector operates on separate memory traces for the left and right ears, but instead suggest that the MMN detector operates on the integrated representation of the two stimuli. Furthermore, the finding that the small frequency distance elicited an MMN in the Frequency-Oddball condition implicates that the absence of an MMN for the same frequency distance in the Location-Oddball condition cannot be attributed to an undetectable frequency distance but instead to the fusion process that abolished the detectable difference between standard and deviant.

As mentioned before, the above chance level localization performance observed in experiment 1 might be due to imperfect fusion. At this point, one might raise the question how such "imperfect" fusion can be explained. In fact, there are several possible explanations. First, one might argue that the fusion process itself is an all-or-nothing process that, however, does not operate on all trials. Second, it might be that for some participants, fusion is stronger than for other ones. Third, fusion itself could be thought of as a gradual process whose extent or degree is determined by stimulus similarity. Whichever of these explanations accounts for the imperfect fusion, aggregating $2 \mathrm{AFC}$ data over trials and participants would yield the observed localization performance.

Furthermore, one might ask whether other factors beside stimulus similarity mediate the fusion of dichotically presented auditory stimuli. One possible factor that might be relevant in this regard is the harmonic relationship between stimuli. There is some evidence that the auditory system can integrate tones into a musical entity even when they are presented to different ears (Hall \& Hess, 1984; Itoh, Miyazaki, \& Nakada, 2003). Accordingly, one could argue that not only the sheer frequency difference between two dichotically presented tones is crucial for fusion, but also the specific frequency ratio of this difference. In the study of Praamstra and Stegeman (1992), the pure tones $(440 \mathrm{~Hz}+880 \mathrm{~Hz})$ formed a strong harmony, namely a perfect octave, whereas the stimuli $(500 \mathrm{~Hz}+700 \mathrm{~Hz})$ in experiment 1 of the present study formed a strong disharmony, called Huygens' Tritone. If the harmonic relationship constitutes a crucial factor for the fusion process, one might expect that fusion is facilitated by a harmonic relationship whereas it is inhibited by a disharmonic relationship. The results of Praamstra and Stegeman and the findings of the present study do not support this hypothesis. This is because Praamstra and Stegeman reported a reliable MMN for deviant location switches of stimuli forming an octave, whereas we observed only a small MMN, no RSE, and a poor localization performance for deviant location switches of tones forming a tritone. Thus, a harmonic relationship of dichotically presented pure tones is not a necessary requirement for fusing tones.

Finally, the results of the present study can be well integrated in recent models explaining the RSE. For example, the parallel grains model assumes that the representation of a stimulus activates a large number of functionally distinct grains (Miller \& Ulrich, 2003). Following stimulus onset, each grain within this pool of grains becomes activated with a certain probability and its activation is transmitted after a random delay to a central decision center. As soon as the number of transmitted grains reaches a criterion, the response is initiated. In redundant trials, each stimulus activates its own pool, and the information of all grains is transmitted to the central center. The more grains are transmitted, the earlier the criterion number will be attained according to the principle of statistical facilitation (cf. Miller \& Ulrich, 2003). According to this model, an RSE results since the number of activated grains is usually higher in redundant than in single-stimulus trials. However, if the grain pools of the redundant stimuli do largely overlap, no RSE will occur (see Schröter et al., 2009). 
With respect to the present study, the absence of an RSE for redundant stimuli with small frequency distances suggests that almost the same number of grains was activated whether two pure tones or only one tone were presented. The absence of a reliable MMN in the small frequency distance conditions is consistent with this notion and provides additional evidence that the number of distinct grain pools is associated with the number of stimulus representations rather than with the number of physical stimuli. Furthermore, it is conceivable that the information in sensory memory is based on these grain pools. Accordingly, the mismatch detector operates on the activated grain pools. As these grain pools do not include location information about the single tones in the small frequency distance conditions, the mismatch detector will not be activated by a deviant location change. Thus, the parallel grains model provides a plausible framework to integrate behavioral and psychophysiological results.

To summarize, the present study provides evidence for the idea that two dichotically presented pure tones can be fused into a single percept and this can prevent the occurrence of an RSE. This fusion process, however, seems not to be an all-or-nothing process since it is crucially influenced by the physical difference between stimuli. Moreover, and importantly, the present results suggest that the fusion of dichotically presented pure tones occurs before or at the level of sensory memory, and thus at early stages of auditory information processing. In conclusion, the present findings extend the results of the study by Schröter et al. (2007) and support the notion that the RSE depends on the number of percepts rather than on the number of physical stimuli.

Acknowledgements This study was supported by the Deutsche Forschungsgemeinschaft (SCHR 1180/2-1). We thank Hartmut Leuthold and three anonymous reviewers for helpful and constructive comments on an earlier version of this manuscript.

\section{References}

Alho, K., \& Sinervo, N. (1997). Preattentive processing of complex sounds in the human brain. Neuroscience Letters, 233, 33-36. doi:10.1016/S0304-3940(97)00620-4

Allen, J., Kraus, N., \& Bradlow, A. (2000). Neural representation of consciously imperceptible speech sound differences. Perception \& Psychophysics, 62, 1383-1393.

Biederman, I., \& Checkosky, S. F. (1970). Processing redundant information. Journal of Experimental Psychology, 83, 486-490. doi:10.1037/h0028841

Bucur, B., Allen, P. A., Sanders, R. E., Ruthruff, E., \& Murphy, M. D. (2005). Redundancy gain and coactivation in bimodal detection: Evidence for the preservation of coactive processing in older adults. Journal of Gerontology, $60,279-282$.
Corballis, M. C. (2002). Hemispheric interactions in simple reaction time. Neuropsychologia, 40, 423-434. doi:10.1016/S0028-3932 (01)00097-5

Cousineau, D. (2005). Confidence intervals in within-subject designs: A simpler solution to Loftus and Masson's method. Tutorials in Quantitative Methods for Psychology, 1, 42-45.

Deacon, D., Nousak, J. M., Pilotti, M., Ritter, W., \& Yang, C. M. (1998). Automatic change detection: Does the auditory system use representations of individual stimulus features or gestalts? Psychophysiology, 35, 413-419. doi:10.1017/S004857729896053X

Deouell, L. Y., Bentin, S., \& Giard, M.-H. (1998). Mismatch negativity in dichotic listening: Evidence for interhemispheric differences and multiple generators. Psychophysiology, 35, 355365. doi:10.1017/S0048577298970287

Diederich, A., \& Colonius, H. (2004). Bimodal and trimodal multisensory enhancement of reaction time: Effects of stimulus onset and intensity. Perception \& Psychophysics, 66, 1388-1404.

Fischer, R., \& Miller, J. (2008). Differential redundancy gain in onset detection versus offset detection. Perception \& Psychophysics, 70, 431-436. doi:10.3758/PP.70.3.431

Gomes, H., Ritter, W., \& Vaughan, H. (1995). The nature of preattentive storage in the auditory system. Journal of Cognitive Neuroscience, 7, 81-94. doi:10.1162/jocn.1995.7.1.81

Gratton, G., Coles, M. G., \& Donchin, E. (1983). A new method for off-line removal of ocular artifact. Electroencephalography and Clinical Neurophysiology, 55, 468-484. doi:10.1016/0013-4694 (83)90135-9

Grimm, S., \& Schröger, E. (2007). The processing of frequency deviations within sounds: Evidence for the predictive nature of the mismatch negativity (MMN) system. Restorative Neurology and Neuroscience, 25, 241-249.

Hall, D. E., \& Hess, J. T. (1984). Perception of musical interval tuning. Music Perception, 2, 166-195.

Horvath, J., Czigler, I., Jacobsen, T., Maess, B., Schröger, E., \& Winkler, I. (2008). MMN or no MMN: No magnitude of deviance effect on the MMN amplitude. Psychophysiology, 45, 60-69.

Ille, N., Berg, P., \& Scherg, M. (2002). Artifact correction of the ongoing EEG using spatial filters based on artifact and brain signal topographies. Journal of Clinical Neurophysiology, 19, $113-124$.

Itoh, K., Miyazaki, K., \& Nakada, T. (2003). Ear advantage and consonance of dichotic pitch intervals in absolute-pitch processors. Brain and Cognition, 53, 464-471. doi:10.1016/S0278-2626 (03)00236-7

Jääskeläinen, I. P., Ahveninen, J., Bonmassar, G., Dale, A. M., Ilmoniemi, R. J., Levänen, S., et al. (2004). Human posterior auditory cortex gates novel sounds to consciousness. Proceedings of the National Academy of Sciences, 101, 6809-6814.

Jacobsen, T., \& Schröger, E. (2001). Is there pre-attentive memorybased comparison of pitch? Psychophysiology, 38, 723-727. doi:10.1111/1469-8986.3840723

Kujala, T., Tervaniemi, M., \& Schröger, E. (2007). The mismatch negativity in cognitive and clinical neuroscience: Theoretical and methodological considerations. Biological Psychology, 74, 1-19. doi:10.1016/j.biopsycho.2006.06.001

Leakey, D. M., Sayers, B. M., \& Cherry, C. (1958). Binaural fusion of low- and high-frequency sounds. The Journal of the Acoustical Society of America, 30, 222-223. doi:10.1121/1.1909549

Macmillan, N. A., \& Creelman, C. D. (2005). Detection theory: A user's guide (2nd ed.). Cambridge: Cambridge University Press.

McKenzie, D. N., \& Barry, R. J. (2006). The independence of memory traces of attended and unattended stimuli. Cerebral Cortex, 16, 1566-1570. doi:10.1093/cercor/bhj093

Miller, J. (1982). Divided attention: Evidence for coactivation with redundant signals. Cognitive Psychology, 14, 247-279. doi:10.1016/0010-0285(82)90010-X 
Miller, J., Patterson, T., \& Ulrich, R. (1998). A jackknife-based method for measuring LRP onset latency differences. Psychophysiology, 35, 99-115.

Miller, J., \& Ulrich, R. (2003). Simple reaction time and statistical facilitation: A parallel grains model. Cognitive Psychology, 46, 101-151. doi:10.1016/S0010-0285(02)00517-0

Molholm, S., Gomes, H., Lobosco, J., Deacon, D., \& Ritter, W. (2004). Feature versus gestalt representation of stimuli in the mismatch negativity system of 7- to 9-year-old children. Psychophysiology, 41, 385-393. doi:10.1111/j.1469-8986.2004.00168.x

Mordkoff, J. T., \& Yantis, S. (1991). An interactive race model of divided attention. Journal of Experimental Psychology: Human Perception and Performance, 17, 520-538. doi:10.1037/00961523.17.2.520

Mordkoff, J. T., \& Yantis, S. (1993). Dividing attention between color and shape: Evidence of coactivation. Perception \& Psychophysics, 53, 357-366.

Näätänen, R. (1995). The mismatch negativity: A powerful tool for cognitive neuroscience. Ear and Hearing, 16, 6-18.

Näätänen, R. (2001). The perception of speech sounds by the human brain as reflected by the mismatch negativity (MMN) and its magnetic equivalent (MMNm). Psychophysiology, 38, 1-21. doi:10.1017/S0048577201000208

Näätänen, R., Paavilainen, P., Rinne, T., \& Alho, K. (2007). The mismatch negativity (MMN) in basic research of central auditory processing: A review. Clinical Neurophysiology, 118, 25442590. doi:10.1016/j.clinph.2007.04.026

Näätänen, R., Tiitinen, H., Jiang, D., \& Alho, K. (1993). Attention and mismatch negativity. Psychophysiology, 30, 436-450. doi:10.1111/j.1469-8986.1993.tb02067.x

Neuloh, G., \& Curio, G. (2004). Does familiarity facilitate the cortical processing of music sounds? NeuroReport, 15, 2471-2475. doi:10.1097/00001756-200411150-00008

Novitzki, N., Tervaniemi, M., Huotilainen, M., \& Näätänen, R. (2004). Frequency discrimination at different frequency levels as indexed by electrophysiological and behavioural measures. Cognitive Brain Research, 20, 26-36.

Odenthal, D. W. (1961). Simultaneous dichotic frequency discrimination. The Journal of the Acoustical Society of America, 33, $357-$ 358. doi:10.1121/1.1908661

Odenthal, D. W. (1963). Perception and neural representation of simultaneous pure tone stimuli. Acta Physiologica et Pharmacologica Neerlandica, 12, 453-496.

Oldfield, R. C. (1971). The assessment and analysis of handedness: The Edinburgh inventory. Neuropsychologia, 9, 97-113. doi:10.1016/0028-3932(71)90067-4

Paavilainen, P., Tiitinen, H., Alho, K., \& Näätänen, R. (1993). Mismatch negativity to slight pitch changes outside strong attentional focus. Biological Psychology, 37, 23-41. doi:10.1016/0301-0511(93)90025-4

Praamstra, P., \& Stegeman, D. F. (1992). On the possibility of independent activation of bilateral mismatch negativity (MMN) generators. Electroencephalography and Clinical Neurophysiology, 82, 67-80. doi:10.1016/0013-4694(92)90184-J

Ritter, W., Deacon, D., Gomes, H., Javitt, D. C., \& Vaughan, H. G., Jr. (1995). The mismatch negativity of event-related potentials as a probe of transient auditory memory: A review. Ear and Hearing, $16,52-67$.
Ritter, W., Sussman, E., \& Molholm, S. (2000). Evidence that the mismatch negativity system works on the basis of objects. NeuroReport, 11, 61-63. doi:10.1097/00001756-20000117000012

Schairer, K. S., Gould, H. J., \& Pousson, M. A. (2001). Source generators of mismatch negativity to multiple deviant stimulus types. Brain Topography, 14, 117-130.

Schröger, E. (1998). Measurement and interpretation of the mismatch negativity. Behavior Research Methods, Instruments, \& Computers, 30, 131-145.

Schröger, E. (2007). Mismatch negativity: A microphone into auditory memory. Journal of Psychophysiology, 21, 138-146. doi:10.1027/0269-8803.21.34.138

Schröter, H., Frei, L. S., Ulrich, R., \& Miller, J. O. (2009). The auditory redundant signals effect: An influence of number of stimuli or number of percepts? Attention, Perception, \& Psychophysics, 71, 1375-1384. doi:10.3758/APP.71.6.1375

Schröter, H., Ulrich, R., \& Miller, J. (2007). Effects of redundant auditory stimuli on reaction time. Psychonomic Bulletin \& Review, 14, 39-44.

Sussman, E. S. (2007). A new view on the MMN and attention debate: The role of context in processing auditory events. Journal of Psychophysiology, 21, 164-175. doi:10.1027/0269-8803.21.34.164

Sussman, E., Gomes, H., Nousak, J. M., Ritter, W., \& Vaughan, H. G., Jr. (1998). Feature conjunctions and auditory sensory memory. Brain Research, 793, 95-102. doi:10.1016/S0006-8993(98)00164-4

Takegata, R., \& Morotomi, T. (1999). Integrated neural representation of sound and temporal features in human auditory sensory memory: An event-related potential study. Neuroscience Letters, 274, 207-210. doi:10.1016/S0304-3940(99)00711-9

Ulrich, R., \& Miller, J. (2001). Using the jackknife-based scoring method for measuring LRP onset effects in factorial designs. Psychophysiology, 38, 816-827. doi:10.1017/S0048577201000610

Ulrich, R., Miller, J. O., \& Schröter, H. (2007). Testing the race model inequality: An algorithm and computer programs. Behavior Research Methods, 39, 291-302.

van Zuijen, T. L., Simoens, V. L., Paavilainen, P., Näätänen, R., \& Tervaniemi, M. (2006). Implicit, intuitive, and explicit knowledge of abstract regularities in a sound sequence: An eventrelated brain potential study. Journal of Cognitive Neuroscience, 18, 1292-1303. doi:10.1162/jocn.2006.18.8.1292

Ward, W. D. (1970). Musical Perception. In J. V. Tobias (Ed.), Foundations of modern auditory theory (Vol. 1) (pp. 405-447). New York: Academic Press.

Winer, B. J. (1971). Statistical principles in experimental design. New York: McGraw-Hill.

Winkler, I. (2007). Interpreting the mismatch negativity (MMN). Journal of Psychophysiology, 21, 147-163. doi:10.1027/02698803.21.34.147

Woldorff, M. G., Hackley, S. A., \& Hillyard, S. A. (1991). The effects of channel-selective attention on the mismatch negativity wave elicited by deviant tones. Psychophysiology, 28, 30-42. doi:10.1111/j.1469-8986.1991.tb03384.x

Woldorff, M. G., Hillyard, S. A., Gallen, C. C., Hampson, S. R., \& Bloom, F. E. (1998). Magnetoencephalographic recordings demonstrate attentional modulation of mismatch-related neural activity in human auditory cortex. Psychophysiology, 35, 283292. doi:10.1017/S0048577298961601 